\title{
Geosynchronous Imaging Fourier Transform Spectrometer (GIFTS): Imaging and Tracking Capability
}

\author{
D. K. Zhou*, A. M. Larar, Xu Liu, and R. A. Reisse \\ NASA Langley Research Center \\ Hampton, VA 23681, USA
}

\author{
W. L. Smith, and H. E. Revercomb \\ University of Wisconsin - Madison \\ Madison, WI 53706, USA
}

\author{
G. E. Bingham, L. J. Zollinger, and J. J. Tansock \\ Space Dynamics Lab., Utah State University, \\ Logan, UT 84322, USA \\ Ronald J. Huppi \\ Ronald J. Huppi, Consultant \\ 6 Fairlane Terrace, Winchester, MA 01890, USA
}

\begin{abstract}
The geosynchronous-imaging Fourier transform spectrometer (GIFTS) engineering demonstration unit (EDU) is an imaging infrared spectrometer designed for atmospheric soundings. It measures the infrared spectrum in two spectral bands (14.6 to $8.8 \mu \mathrm{m}, 6.0$ to $4.4 \mu \mathrm{m})$ using two $128 \times 128$ detector arrays with a spectral resolution of $0.57 \mathrm{~cm}^{-1}$ with a scan duration of $\sim 11$ seconds. From a geosynchronous orbit, the instrument will have the capability of taking successive measurements of such data to scan desired regions of the globe, from which atmospheric status, cloud parameters, wind field profiles, and other derived products can be retrieved. The GIFTS EDU provides a flexible and accurate testbed for the new challenges of the emerging hyperspectral era. The EDU ground-based measurement experiment, held in Logan, Utah during September 2006, demonstrated its extensive capabilities and potential for geosynchronous and other applications (e.g., Earth observing environmental measurements). This paper addresses the experiment objectives and overall performance of the sensor system with a focus on the GIFTS EDU imaging capability and proof of the GIFTS measurement concept.
\end{abstract}

Keywords-image; remote sensing; satellite; Fourier transform spectrometer (FTS); Michelson interferometer;

\section{INTRODUCTION}

Severe weather (e.g., hurricanes, tornadoes, floods, ice storms, etc.) impacts our daily lives. An accurate weather forecast is needed now more than ever for planning daily activities and for optimizing the preparation for the onset of life-threatening and property-damaging weather (e.g., a hurricane landfall). The improvement of weather prediction requires high resolution temporal and spatial measurements of temperature, moisture, and wind profiles. High density and timely thermodynamic profile measurements are needed to forecast where and when severe convective storms will form before they are visible on radar or in satellite cloud imagery. Quasi-continuous measurements of the moisture flux are needed for timely forecasts of storm intensity changes.
Atmospheric wind profiles are needed to predict the storm's trajectory, and in the case of tropical storms and hurricanes, their landfall location and time. Based on these considerations, the GIFTS measurement concept [1] has been developed within the reach of new technologies for improving weather prediction accuracy.

The GIFTS EDU utilizes the advantages of cryogenic FTS technology developed by Stewart Radiance Laboratory (SRL) and Space Dynamics Laboratory (SDL) to improve sensitivity and calibration stability [2]. The EDU has undergone a series of thermal vacuum tests followed by an extensive calibration phase. The success of the GIFTS EDU is not only critical to the GIFTS program itself but also for a demonstration of the new technologies (e.g., large area format LWIR FPA and high speed detector readout electronics, mini-pulse tube cooler, lightweight carbon composite optics, long lifetime precision laser, etc.) incorporated in the EDU. A ground-based GIFTS EDU measurement experiment was conducted during September 2006 to collect the real atmospheric, lunar, and mountain-sky data needed to characterize and demonstrate the sensor system. This experiment is critical in demonstrating the capabilities of the instrument, its calibration, and its associated data processing procedures including the retrieval of scientific products. An overview of this experiment is presented with some preliminary analyses to illustrate that a successful experiment has been achieved and to reveal the GIFTS sensor system and measurement concept.

\section{GIFTS EDU INSTRUMENTATION}

A detailed description of the GIFTS EDU instrumentation is provided by Elwell et al. [3]. A diagram of the optical system is shown in Figure 1. The pointing mirror and off-axis telescope (M1, M2, and M3) are made of high thermal conductivity silicon carbide to minimize solar radiation effects and reduce mass. The telescope baffle cover, which would provide a contamination seal for the optics during launch, orbital insertion and extended thruster firings, was not

\footnotetext{
*daniel.k.zhou@nasa.gov; phone: 757-864-5663; fax: 757-864-7790
} 
implemented in the EDU. The two-axis scene selection mirror, although not implemented in the EDU, is specified to provide a field-of-regard (FOR) of $\pm 12.9^{\circ}$ in both the cross and vertical Earth directions, allowing clear space views on all sides of the globe. Information on other sub-systems, such as Laser metrology, Fourier transform spectrometer (FTS), telescope and relay optics, aft optics/electronics, mechanical cooler, visible camera, and on-board calibration sources, are described and found in Elwell et al. [3].

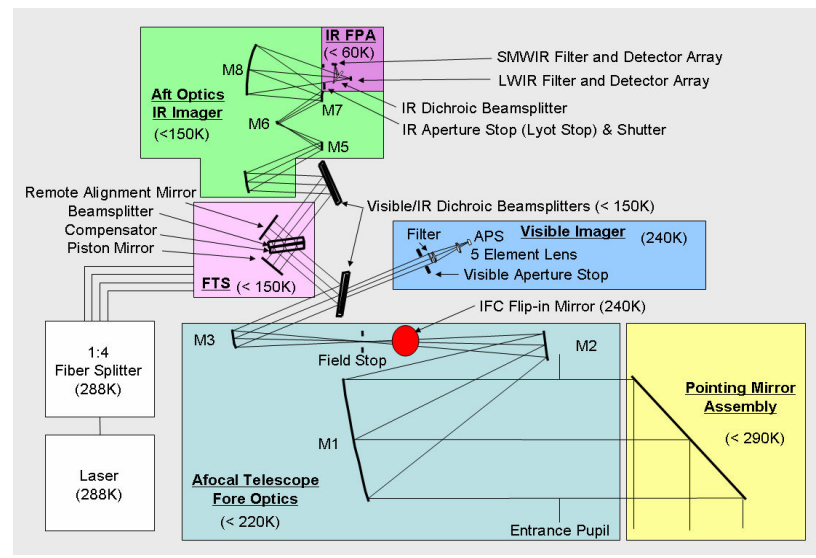

Figure 1. A conceptual optical system ray trace for the GIFTS sensor.

\section{GIFTS EDU GROUNG-BASE MEASUREMENT EXPERIMENT}

The primary objectives of this experiment are to assess instrumental performance, to produce measured spectral radiance data utilizing the GIFTS EDU, to retrieve atmospheric conditions from the radiance data, and to validate the GIFTS EDU data products at all stages with ancillary data collected coincidently. A secondary objective was to demonstrate the capability of monitoring atmospheric spatial and temporal variations, an important aspect of the GIFTS measurement concept. In addition, a series of events was planned to characterize the sensor system such as the presence and levels of vibration and optical jitter, and their affect on system performance and data products.

\section{A. Experiment objectives and setup}

A series of 8 science and engineering data collection events was carefully planned to achieve the previously defined objectives of this experiment. The events included a CrossCheck Event (CCE), a Moon Viewing Event (MVE), a Moon Tracking Event (MTE), an Atmospheric Variation Event (AVE), an Outgas Monitoring Event (OME), a Moon-tracking and Sky-viewing Event (MSE), a Vibration Test Event (VTE), and a Jitter Test Event (JTE). Each event had its own objectives. Quick look displays for the GIFTS EDU and two Atmospheric Emitted Radiance Interferometer (AERI) instruments provided on-site scientists and engineers real time feed back to ensure the success of the experiment.

Prior to the science data collection, the GIFTS EDU was checked out and made ready to collect quantified science data. Since this was a ground-based experiment, a Zinc Selenide
( $\mathrm{ZnSe}$ ) window was used to keep the GIFTS EDU under a space-simulated thermal vacuum condition in a chamber. This window is not needed when GIFTS is in space. Calibration data of the FTS sensor viewing through the ZnSe window were collected and analyzed prior to the science data collection events; these data are to be used to calibrate GIFTS including the window and understand the window effects on the sensor performance relevant to the science data.

\section{B. Spectral imaging capability}

One of the fundamental advantages of the GIFTS concept is illustrated by the spectral images shown in Figure 4, an example of the lunar/atmospheric images obtained with two GIFTS infrared detector arrays indicating that the lunar/atmosphere image is a function of wavenumber. Lunar and sky measurements were obtained during the GIFTS ground-based measurement experiment; the Moon Tracking Event (MTE) was conducted on 11 September 2006.

The major objective was to obtain high quality images of the moon throughout GIFTS EDU spectral channels at the highest spectral resolution of $0.57 \mathrm{~cm}^{-1}$ while tracking the moon so as to demonstrate the GIFTS hyperspectral resolution imaging capability. The measurements occurred at approximately 4:52 a.m. Mountain Daylight Time (MDT), 11 September 2006, in North Logan, Utah. The radiance spectra observed by detector elements viewing the warm lunar surface (e.g., the red spectrum) are similar in spectral character to those that will be obtained by GIFTS viewing the Earth from orbit. The radiance spectra observed by detector elements viewing the cold space background (e.g., the blue spectrum) are inverted relative to those observed by GIFTS viewing the warm lunar surface background (i.e., the red spectrum). In spectral regions where the atmosphere is a very strong absorber/emitter, one sees little difference between the lunar and cold space background views. In other words, a threedimensional (3-d) variation (i.e., 2-d in spatial and 1-d in spectral variation) of atmosphere and lunar surface was captured with one scan ( $\sim 11$ seconds). The power of the GIFTS concept of hyperspectral information at each spatial location in the image is illustrated.
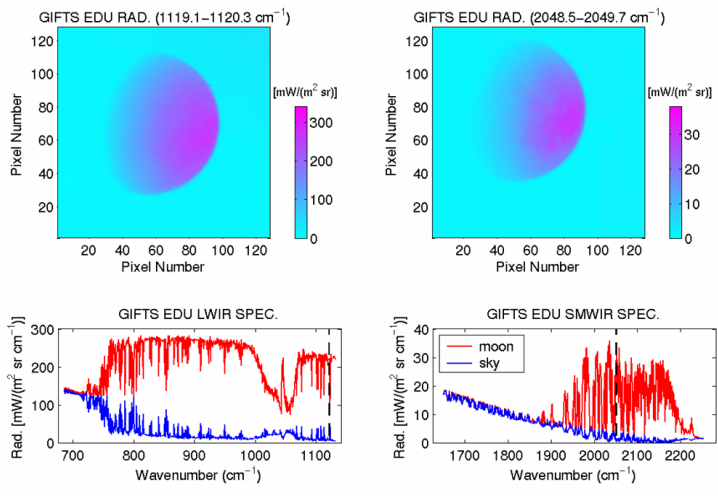

Figure 2. Illustrations of lunar and atmospheric measurements provided by the GIFTS EDU on 11 September 2006 - a demonstration of the GIFTS imaging spectrometer measurement ability. 


\section{Image-tracking capability}

Furthermore, GIFTS is a geostationary imaging spectrometer, providing radiance observations in four dimensions (i.e., 2-d in horizontal-spatial, 1-d in spectral variation, and $1-d$ in temporal variation). The revolutionary aspect of GIFTS is that it provides access to the vertical dimension of the wind field with more accurate altitude assignment than possible with current systems. With the $4^{\text {th }}$ dimension of temporal variation, the vertical profiles of wind velocity can be achieved by tracking the movement of smallscale, non-condensed moisture features retrieved with first 3-d atmospheric variations. An algorithm to derive altituderesolved atmospheric motion vectors (AMV) is being developed and evaluated using simulated GIFTS instrument data [4]. As the GIFTS is designed for geosynchronous orbit, the field of view from the ground to monitor moisture variation is limited. To demonstrate GIFTS 4-d observation ability with the GIFTS EDU on the ground, an experiment called Outgas Monitoring Event (OME) is designed to monitor both temporal and spatial variations of the atmosphere with the GIFTS EDU when Sulfur Hexafluoride $\left(\mathrm{SF}_{6}\right)$ gas is released in the near field. The experiment was conducted on 18 September 2006, at approximately 5:30 p.m. MDT. The $\mathrm{SF}_{6}$ gas was released at a flow rate of 5 Liters/min from a quarter inch pipe. The GIFTS EDU was at a distance of $\sim 70 \mathrm{ft}$ from the pipe where the GIFTS field of view about $1 \times 1 \mathrm{ft}^{2}$ was provided. The spectral signature of $\mathrm{SF}_{6}$ gas emission was clearly evident as detected by both GIFTS and AERI.

During this experiment, a set of 35-consective scans with a high spectral resolution $\left(0.57 \mathrm{~cm}^{-1}\right.$; with a scan duration of $\sim 11$ sec.), $\mathrm{SF}_{6}$ gas was released from the top of the images shown in Figure 3 during scans 6-30. The spatial, spectral, and temporal distributions of $\mathrm{SF}_{6}$ infrared emissions were evidently captured by the GIFTS EDU demonstrating GIFTS observation concepts such as moisture traced wind measurements. Distribution of $\mathrm{SF}_{6}$ infrared emissions shown in Figure 3 is integrated in a spectral region of $925-951 \mathrm{~cm}^{-1}$.

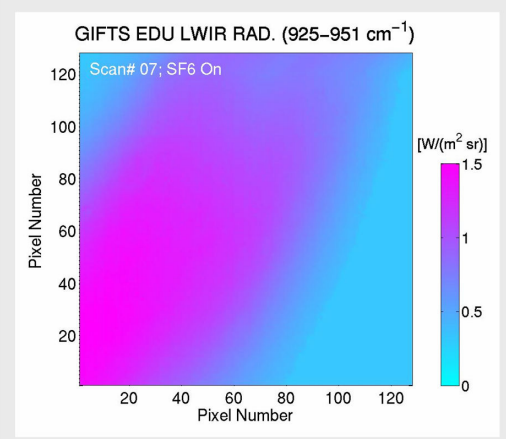

Figure 3. Illustrations of SF6 outgas measurements provided by the GIFTS EDU on 18 September 2006 - a demonstration of the GIFTS image tracking ability.

\section{CONCLUSION}

The GIFTS new technologies have been integrated into the GIFTS EDU. The GIFTS EDU ground-based measurement experiment was carefully planned and successfully conducted. The GIFTS measurement capabilities and proof of concept were successfully demonstrated with the data collected during ground-based measurements and the thermal-vacuum performance and calibration tests. Preliminary analyses of atmospheric science data validate the GIFTS design and capability to provide science data from a space-borne system. Further data analyses will improve our understanding of the measurement characteristics of the GIFTS EDU so as to provide information needed to optimize the sensor design and allow the construction of a high-performance, low-risk, and space flight GIFTS instrument. It also provides a great risk reduction effort for any other instruments similar to GIFTS EDU.

\section{ACKNOWLEDGMENT}

The GIFTS EDU, built and calibrated by the Space Dynamics Laboratory of Utah State University, was a NASA New Millennium Program (NMP) sponsored instrument designed to validate the new technologies. NMP was managed by NASA's Jet Propulsion Laboratory and the GIFTS project was managed by NASA Langley Research Center. The groundbased experiment was supported by the NASA Headquarters and NASA Langley Research Center. The authors acknowledge support from NASA Headquarters Earth Science Division Deputy Director Dr. Bryant Cramer. The authors express their sincere thanks to the GIFTS team members from various organizations. The personnel who contributed to this project are too numerous to mention by name, nonetheless their personal contributions are greatly appreciated.

\section{REFERENCES}

[1] W. L. Smith, F. W. Harrison, H. E. Revercomb, and G. E. Bingham, "Geostationary Imaging Fourier Transform Spectrometer (GIFTS) - The New Millennium Earth Observing-3 Mission," in Current Problems in Atmospheric Radiation, M. L. Smith, and Y. M Timofeyev, Eds., Proc of IRS 2000, A Deepak Publishing, Hampton, Virginia, 81-84 (2001).

[2] R. J. Huppi, A. J. Steed, and E. R. Huppi, "Cryogenically cooled Fourier transform spectrometers," in International Conference on Fourier Transform Infrared Spectroscopy, H. S. Bellingham, Ed., Proc. SPIE 289, 345-354 (1981)

[3] J. D. Elwell, and co-authors, "A Geosynchronous Imaging Fourier Transform Spectrometer (GIFTS) for Hyperspectral Atmospheric Remote Sensing: Instrument Overview \& Preliminary Performance Results," in Infrared Spaceborne Remote Sensing XIV, M. Strojnik, Ed., Proc. SPIE 6297, 62970S (2006)..

[4] C. Velden, G. Dengel, R. Dengel, A. H. Huang, D. Stettner, H. Revercomb, R. Knuteson, and W. Smith, Sr., "Determination of wind vectors by tracking features on sequential moisture analyses derived from hyperspectral IR satellite soundings," preprints, $13^{\text {th }}$ AMS Conf. on Satellite Meteor. And Ocean., Amer. Meteor. Soc. (2004). 